\title{
ECG Auto-analyzing System on Android for 3AHcare Node
}

\author{
Huan Jin ${ }^{1,2}$, Zhen Fang ${ }^{1}$, Xianxiang Chen ${ }^{1}$, Zhan Zhao ${ }^{1}$, ZhiHong Xu ${ }^{1,2}$, DongDong Lou ${ }^{1,2}$, XingZu Guo ${ }^{1,2}$ \\ ${ }^{1}$ Institute of Electronics, Chinese Academy of Sciences, BeiJing, 100190, China \\ ${ }^{2}$ University of Chinese Academy of Sciences, BeiJing, 100049, China \\ muye0622@gmail.com, \{zfang, zhaozhan\}@mail.ie.ac.cn
}

\begin{abstract}
ECG (Electrocardiograph) is the primary method for cardiovascular disease detection. The main purpose of this research work is to develop an ECG auto-analyzing system on Android platform for 3AHcare Node. 3AHcare Node is a health monitoring device developed by IECAS, and ECG auto-analyzing system analyzes the ECG signal detected by 3 AHcare Node in real time. The ECG auto-analyzing system consists of two parts: the detection part and the diagnose part. In the detection part, the noise is eliminated first, and then the feature parameters, such as R peak, RR interval, QRS width and RR rhythm etc are extracted. The diagnose part is used to classify the ECG signal into three categories: the PVC (Premature Ventricular Contraction), NORMAL and UNKNOWN, at last, give primary diagnose suggestions for users. The ECG autoanalyzing system was first measured with data from MIT/BIH Arrhythmia Database, and then modified and tested by ECG data detected with our 3AHcare Node.

Index Terms - ECG, Android, 3AHcare Node, ECG analyzing, ECG classification.
\end{abstract}

\section{Introduction}

Cardiovascular disease has the highest death rate globally which means that much more people die from it annually than any other diseases. In 2012, the total number of cardiovascular patients in China is 230 million, and near 3.5 million people died every year in average. According to the World Health Organization, in the year 2030, it is estimated that 25 million people will die because of cardiovascular disease, especially caused by heart attack and stroke.

For many years, ECG has been the best method for detecting cardiovascular diseases. But the traditional way of measuring ECG is confined to hospital. According to Beijing Emergency Centre, more than $71 \%$ cardiac patients die at home or office, majority of them lose the best time for rescue and die outside the hospital. Because of the unpredictability and suddenness of the heart diseases, and in order to offer rescue opportunities for heart patients, many institutes devote themselves to home-use ECG measuring devices. But there are still many kinds of drawbacks needed to be overcome.

The earliest home-use ECG monitoring devices require the patients to detect ECG data in quiet. These kinds of devices lack the convenience of moving, and have worse antiinterference ability.

Some devices such as NUVANT MCT System of Corventis Company, Heartline of Aerotel Company, can be used at anytime without influencing patients' daily activities, but they do not offer associated analyzing system. It still has to turn to doctors for diagnose information. The famous Holter
System includes a small monitoring device and an analysing system, but the ECG analysing softwares is $\mathrm{C} / \mathrm{S}$ architecture on personal computer, which can't work at anytime in need, for instance, patients cannot use the ECG analysing softwares when running. Some institutes find out the problems of convenience of ECG analysing softwares, such as iCard on iOS, Cardiograph application on Android, but both of them either have simple functions or are little professional. Several applications on Android developed by some institutes, although they have rich functions, majority of them only tested by the MIT/BIH arrhythmia database, and don't have the confidence of performing well in any other portable ECG monitoring devices.

Compared to the above mentioned projects, our ECG auto-analysing system for 3 AHcare Node has the following main features:

1) Portable. The 3 AHcare Node - a health monitoring device developed and tested by the Institute of Electronics, Chinese Academy of Sciences (IECAS) - is a small and light device. Its size is about $6 \mathrm{~cm}$ long and $5 \mathrm{~cm}$ wide. Patients can test ECG in the common way, or wear it on an affiliated vest as well (Fig. 1). Except for the 3AHcare Node, the ECG autoanalyzing system on Android also can be easily taken.

2) Easily and widely used. We choose the Android platform to develop ECG auto-analyzing system. As statistics given by IDC (Internet Data Center), the number of users of Android platform account for $68 \%$ globally in mobile market. It is to say, most of people use Android platform, and they can easily get start of our ECG auto-analyzing system.

3) Real time. The 3 AHcare Node can communicate with ECG auto-analyzing system in real time. At the same time, the detected data will be presented on the screen, and the relative results will be calculated and shown quickly. While other ECG applications on iOS or Android do not perform well in this aspect. This feature makes our ECG-analyzing system much more outstanding than other ECG analyzing applications.
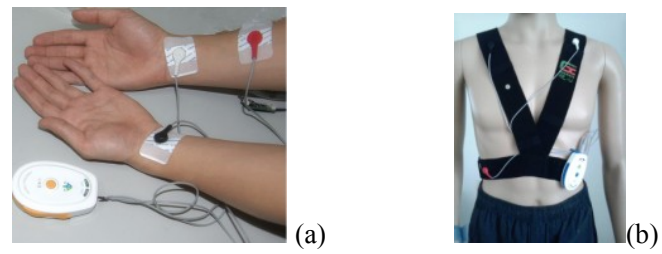

Fig. 1 The two ways to measure ECG signal: (a) The common way; (b) Wear on an affiliated vest. 
4) Online Emergency Detection. ECG auto-analysing system owns the function to connect into the internet or call the caregivers through GPRS. Patients can connect doctors for diagnose information at anytime, or call family members at emergency.

The reminder of the paper is divided as follows: the section 2 will give an introduction of our whole System; the auto-analyzing algorithms will be described in section 3; the results of experiment and our conclusion and future work will be presented in section 4 and section 5 separately.

\section{System Overview}

Our whole system actually includes two parts: the 3AHcare Node and ECG Auto-analyzing System.

\section{A. The 3AHcare Nodes}

For 3AHcare Node, we selected Texas Instruments' $\mathrm{CC} 2530$ as the main control unit, which integrated microcontroller and the radio transceiver function in a chip. A Zigbee connection is established between 3AHcare node and PAD.

The TI's 24 bit ADS1294R was adopted as ECG measurement module in the 3AHcare Node. ADS1294R is a chip of multichannel, simultaneous sampling, 24-bit, deltasigma $(\Delta \Sigma)$ analog-to-digital converters (ADCs) with built-in programmable gain amplifiers (PGAs), internal reference, and an onboard oscillator. Moreover, the ADS1294R also integrates respiration impedance measurement circuit which could be used to monitor respiration directly. The ADS1294R incorporates all of the features that are required in our design of a compact and low-power ECG and respiration monitoring node, and its exceptional performance and high levels of integration make the front-end ECG signal processing circuits very simple and largely reduced the physical size and power consumption of the node (Fig. 2).

With many circuits integrated on a single board, our 3AHcare Node not only has the capacity of detecting ECG, but also owns the ability of measuring multiple other physiological parameters such as respiratory rate, electroencephalograph (EEG), blood oxygenation (SpO2), body temperature, etc.

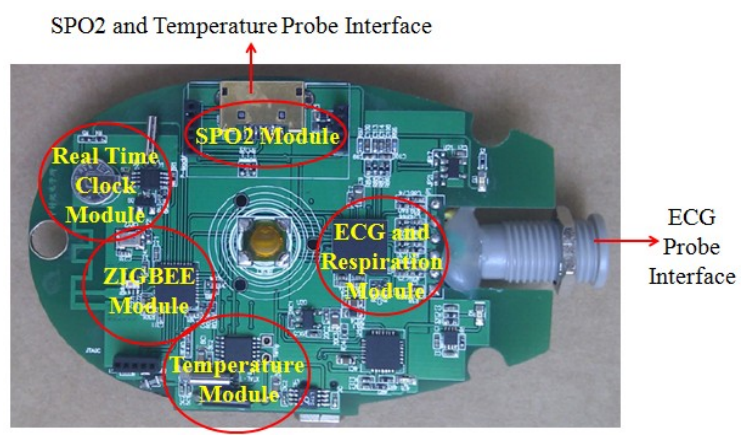

Fig. 2 The IECAS 3AHcare Node

In those physiological parameters, ECG signal is the most important and complex parameter. So in our research, we developed an ECG auto-analyzing system at first.

\section{B. ECG Auto-analyzing System}

ECG Auto-analyzing System has the ability of displaying ECG data in real time, at the same time, positions the R points in the dynamic graph, calculates and displays the value of heart rate, furthermore, classifies the ECG data into right categories, and gives the primary diagnosis suggestions about the heart.

After several investigations, we found out the best architecture for our ECG Auto-analyzing System, as shown in Fig. 3.

The rectangle part of Fig. 3 is the main contents of this research. The ECG auto-analyzing system comprises two parts: the auto detection unit and the auto diagnose unit. The auto detection unit is divided into three modules: noise elimination module; R, RR interval extraction module; other features extraction module. The auto diagnose unit is a classification module actually, which consists of a series of rules. According to the characters of R, RR interval, QRS width and rhythms features, the classification module put the detected data into three categories: Normal, PVC (Premature Ventricular Contraction) and Unknown. At last, give the primary suggestions about the disease.

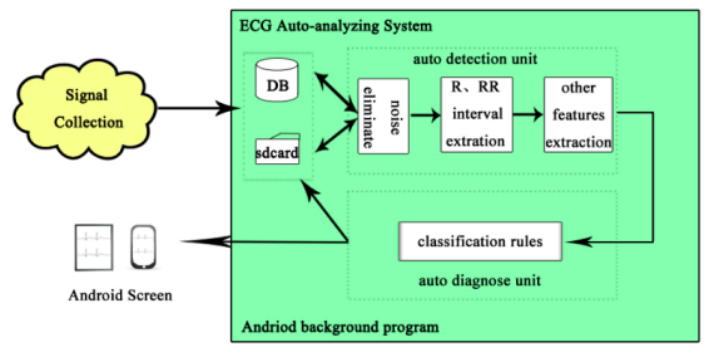

Fig. 3 ECG Auto-analyzing System Architecture.

The detected ECG data is read as data stream, and will be analyzed by ECG auto detection unit in real time. Meantime, the data will be stored as a text in the sqlite database or the sdcard of the Android platform, the name of the text will be set by the time when the signal was detected, and this kind rule of naming is easy for search and can be stored for a long time. Fig. 4 is the working situation of the whole system.

\section{Auto-analyzing Algorithms}

There are many kinds of algorithms to achieve the goal described above, but the algorithms must meet the needs of high efficiency and accuracy. So we choose the algorithms as follows.

\section{A. Sliding window median filter algorithm}

Although the 3AHcare Node owns noise suppression module, but in order to gain data with high quality, we need to deal with noise in auto detection unit once again, especially the baseline shift, which has close relationship with amplitude and slope. We use the sliding window median filter algorithm to eliminate baseline shift. And the other kind noises will be handled in the Improved Pan-Tompkins algorithm. 


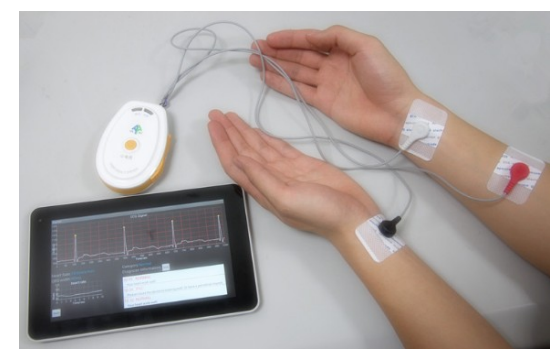

Fig. 4 The working situation of the whole system

The sliding window median filter algorithm is described as follows:

a. Select the appropriate width of the sliding window, which is always set as an odd number.

b. Stretch the both sides of ecg1, and get the ecg2, as in (1). The length of ecg2 is $\mathrm{L}+\mathrm{W}-1$.

$$
\operatorname{ecg} 2(i)=\left\{\begin{array}{c}
\operatorname{ecg} 1(0), 0 \leq i \leq \frac{W-1}{2} \\
\operatorname{ecg} 1\left(i-\frac{W-1}{2}\right), \frac{W-1}{2}<i \leq L+\frac{W+1}{2} \\
\operatorname{ecg} 1(L-1), L+\frac{W-1}{2}<i \leq L+W-2
\end{array}\right.
$$

c. Add the window to ecg2, and use the median filter algorithm on those data in window. The median filter algorithm is a sort algorithm in fact, it is used to calculate the median datum, and the median data compose the BL array, as in (2).

$B L(i)=$ median $[\operatorname{ecg} 2(i): \operatorname{ecg} 2(i+W)], 0 \leq i \leq L-1$.

d. The array ecg1 subtract BL, and the result is ecg3, which is the ECG data without baseline shift.

Fig. 5 is the flow chart of sliding window median filter algorithm.

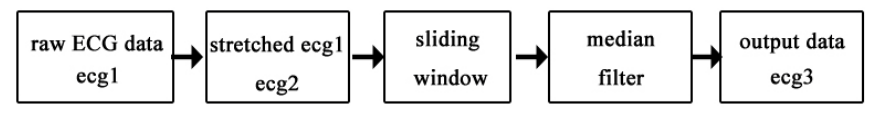

Fig. 5 Sliding window median filter algorithm

\section{B. Improved Pan-Tompkins algorithm}

$R$ and $R R$ interval are two important features of ECG signal. So as to meet the need of efficiency, we use the Improved Pan-Tompkins algorithm. Fig. 6 is the flow chart.

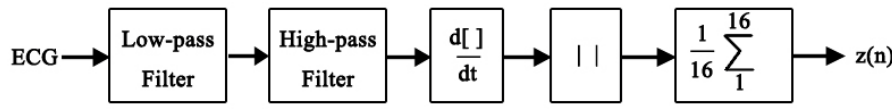

Fig. 6 Improved Pan-Tompkins algorithm

The traditional Pan-Tompkins algorithm consists of lowpass filter, high-pass filter, derivative, square and average value in range of sliding window. There are two differences between the traditional and improved algorithm. One is that we use absolute instead of square, the other one is the length of sliding window, which has close relationship with QRS width. And in principle, the length of sliding window must vary in the range between $80 \mathrm{~ms}$ and $100 \mathrm{~ms}$, according to the standard width of QRS complex.

\section{$\boldsymbol{C}$. The other features extraction algorithm}

In this module, we extract two kinds of features, first is the QRS width, the other one is the RR rhythm.

QRS width refers the width of the QRS complex, which is a fundamental feature used for classifying ECG data. The method to calculate the QRS width is actually locating the onset and offset of QRS complex. The following is the main rules.

a. First we search out from the fiducial point (detection point from the beat detector) until we find a region that precedes the QRS complex and a region that follows the QRS complex where at least 5 samples are within a $100 \mathrm{uV}$ range. This gives us a first estimate of the QRS onset and offset.

b. Find out the maximum and minimum slopes of QRS complex, and remark those points.

c. Search back from the maximum point, with continually comparing with the $1 / 4$ of the maximum slope.

d. Search forward from the minimum point, and continually compare with $1 / 4$ of the minimum slope.

e. Compare the onset and offset with the isoelectric start and end separately. At last, we get the final results of onset and offset.

Because many unexpected tiny noises mix in the signal, we have to estimate the points of onset and offset time after time. And the difference of offset and onset is the QRS width we needed.

The RR rhythm features means the performance of a string of beats, such as normal rhythm, bigeminal rhythm and so on. To analyze RR rhythm, we need to judge the pattern of RR interval first. We marked the RR interval into five features: $\mathrm{NN}, \mathrm{NV}, \mathrm{VN}, \mathrm{VV}$ and QQ. $\mathrm{N}$ means Normal, $\mathrm{V}$ means ventricular, $\mathrm{Q}$ means unknown, and $\mathrm{NN}$ means two consecutive normal beats, $\mathrm{NV}$ means a normal beat followed by a ventricular beat, VN, VV and QQ can be deduced in the same way.

We also use a series of rules to extract RR rhythm features. There are three main rules.

a. The near two RR rhythms are the same if the difference of the two RR rhythms is less than the $1 / 8$ of the sum of them.

b. The current RR interval is considered to be NV if its interval is less than $3 / 4$ of the preceding interval, which is marked as NN type.

c. Calculate the average of NN length in an eight length RR interval buffer, and compared it with current RR interval.

The detailed rules of this algorithm can refer to [4].

\section{Classification algorithm}

After we get the features of R, RR interval and QRS width, there are many ways to classify the ECG signal. For example, the simplest way is comparing these test results of features with the standard results of them. But this kind of rules can only get coarse results, in order to make the results much more trustful, we adopt the algorithm from [4]. 
This classification algorithm has two main steps. First, it will match current beats to former beat templates. If no types were found, it will classify the current beat with its features, and update the beat templates. However, the algorithm compares current beat not only with the former information, but also with the following beats. Combined these two steps, we can classify the data into three categories: Normal, PVC and Unknown.

\section{Experimental Results}

Unlike many other ECG auto-analyzing programs, most of which is developed by Matlab or $\mathrm{C} / \mathrm{C}++$ language, our system is developed with Java language on Android. With the algorithms list above, we succeed in implementing our system and got the ideal results.

In our research, first we tested our system with ECG records from MIT/BIH arrhythmia database. When the consequences matched the categories from annotation text, we modified our ECG auto-analyzing system to meet the need of 3AHcare Node. The main difference between the records from MIT/BIH arrhythmia database and the data measured with 3 AHcare Node is the data type, it is to say, the records of $\mathrm{MIT} / \mathrm{BIH}$ is integer type, and our data is float type, and the integer type will save much more time of analyzing than the float type, what we had to do was making our system work in real time at any type. After either of the data from those two places can be processed by our system, we tested and kept the accuracy rate as obtained before. Now our system can process any data types, which also make our system well expanded.
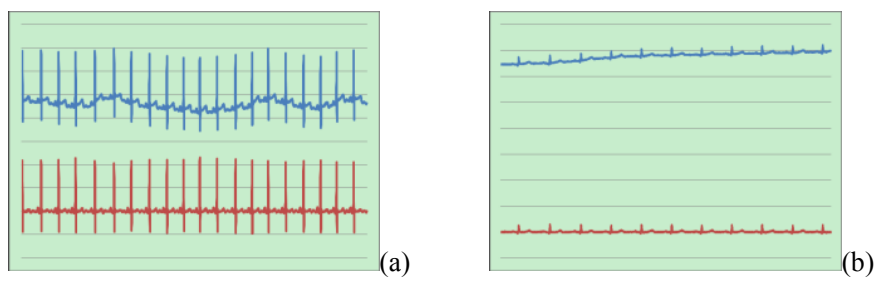

Fig. 7 Baseline shift elimination: (a) a fragment of record 115 from MIT/BIH; (b) data fragment from 3 AHcare Node.

Fig. 7 is the results after baseline shift elimination. Fig. 7(a) is a fragment of record 115 from MIT/BIH, Fig. 7(b) is the data measured by 3 AHcare Node. Upper line is the raw data, and lower line is the result after processed.

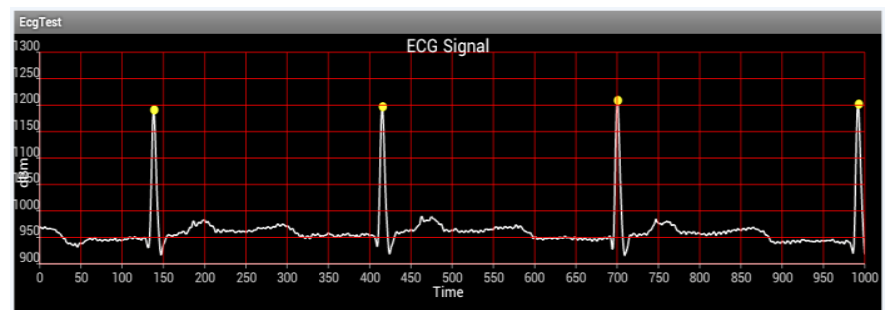

Fig. 8 Locate the R point in the dynamic graph
As shown in Fig. 7, our system can eliminate the baseline shift nearly without lose any important data. And the signal without baseline shift has better user experience. At the same time, the ECG auto-analyzing system will locate the $\mathrm{R}$ in the dynamic graph. Fig. 8 is the screenshot of that dynamic graph. We can see from the graph that the $\mathrm{R}$ peak is accurately pointed out.

Many patients may have little knowledge of reading ECG signal, so our ECG auto-analyzing system translates it for them, as show in Fig. 9. The value of the heart rate is varied according the reality situation of the patient's heart, and the QRS width, the classification are the same. What's more, the analyzing system will give some suggestions about the current situation. In some abnormal cases, the suggestions will be shown in highlight or red color, which is used to attract patients' attention to take measures in time.

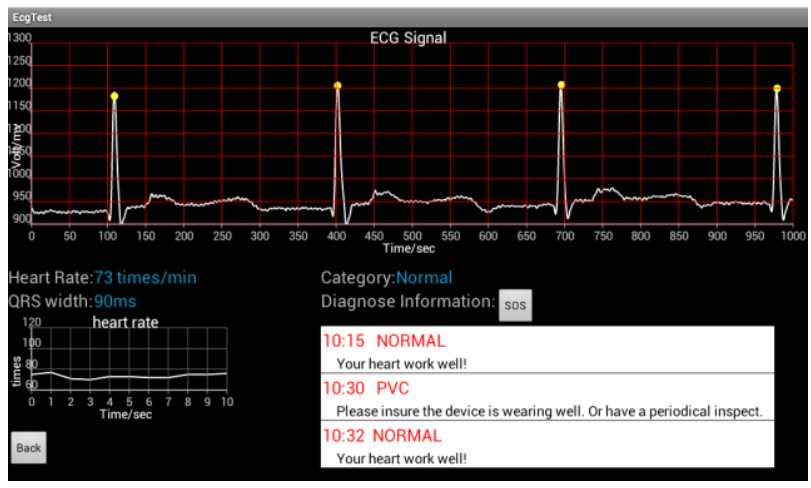

Fig. 9 The diagnose information

\section{Conclusion and Future Work}

Now, anyone can use ECG auto-analyzing system associate with 3 AHcare Node to measure ECG signal. Without the help of doctors, patients can achieve his heart information at anytime in anywhere. The suggestions from ECG autoanalyzing system also help the patients to take measures in time, which will improve the possibility of survive.

But our system only classifies the ECG signal into three categories. In the following research, we will expand our categories with the help from professors of medicine. At the same time, we will add functions to analyze the other physiological parameters.

\section{References}

[1] Z. Fang, Z. Zhao, F.M. Sun, etc "The 3AHcare Node: Health Monitoring Continuously", IEEE Healthcom 2012, pp.365-366, 2012.

[2] J. Pan, W. J. Tompkins, "A Real-Time QRS detection Algorithm", IEEE Trans. Biomed. Eng., vol. 32, no.3, pp. 230-236, 1985.

[3] P. S. Hamilton, Willis J. Tompkins, "Quantitative Investigation of QRS Detection Rules Using the MIT/BIH Arrhythmia Database", IEEE Trans. Biomed. Eng., vol. 33, no.12, pp. 1157-1165, 1985.

[4] P. S. Hamilton, E.P. Limited, "Open source ECG analysis", Computers in Cardiology, pp.101-104, 2002. 